\title{
Adaptation in distance perception with head-movement parallax serving as the veridical cue
}

\author{
HANS WALLACH \\ Swarthmore College, Swarthmore, Pennsylvania 1908I \\ and \\ ANN O'LEARY \\ University of Pennsylvania, Philadelphia, Pennsylvania 19174
}

\begin{abstract}
When head-movement parallax functioned as the sole veridical distance cue during exposure to spectacles that altered the eyes' oculomotor adjustments, sizable adaptation was obtained. This result showed that a discrimination of the distances of 60 and $30 \mathrm{~cm}$ can be based on head-movement parallax. Using adaptation in demonstrating that head-movement parallax can serve as a distance cue circumvents the problem that the presence of accommodation normally presents when such a demonstration is attempted. The usual contamination of head-movement parallax with accommodation is avoided, because accommodation is altered by the spectacles and does not function as a veridical cue along with head-movement parallax.
\end{abstract}

The question has recently been raised whether head-movement parallax (HMP) can serve as a cue for the distance of a single object from the eyes. McConkie and Farber (Note 1) tested for the effectiveness of HMP as a cue for absolute distance by having subjects match the distance of a target observed monocularly during head movements with another target that was given with a complete set of distance cues. They found that their subjects "had some distance information up to about $80 \mathrm{~cm}$." Beyond that point, the matches did not reflect the real target distances. The authors attributed the subjects' performance at distances between 30 and $80 \mathrm{~cm}$ to the effect of accommodation and concluded that movement parallax plays no role in absolute distance perception.'

We had reason to believe that HMP does serve as a cue for short absolute distances. Demonstrating that it does apparently requires a procedure where accomodation is eliminated. It may be done with cycloplegia and a bifocal lens, but would necessitate medical supervision. Instead, we dealt with accommodation indirectly. We demonstrated the effectiveness of HMP as a distance cue by using it as the sole veridical cue in adaptation in distance perception. In this procedure, subjects wore spectacles that forced the eyes to view objects with accommodation and convergence altered so that they misrepresented

This work was supported by Grant BNS75-19095 A01 from the National Science Foundation to Swarthmore College, Hans Wallach, principal investigator. We are most grateful to $\mathrm{V}$. R. Carlson for his help in preparing this report. object distances. Since accomodation did not represent the real distances as did HMP, the two factors did not operate in parallel as they normally do.

Adaptation in distance perception that is based on the cues provided by accommodation and convergence was first reported by Wallach and Frey (1972a). One of the spectacles these authors used altered these oculomotor adjustments so that they represented object distances as shorter than the true distances. They consisted of negative lenses that forced the eyes to accommodate for a shorter distance than the actual target distance would warrant and of wedge prisms that caused the eyes to converge more than the target distance required. Under conditions where these oculomotor adjustments were the only distance cues available, these "near glasses" caused apparent distances to be shortened and the perceived sizes of target objects to be smaller. The latter effect occurred because perceived size is proportional to the product of image size and distance as registered by perceptual processes. Prolonged wearing of such spectacles changed the relation between the oculomotor cues, on the one hand, and perceived distance, that is, apparent and registered distance, on the other, in such a way that apparent distances and sizes gradually became more veridical; a compensation for the effect of the spectacles developed. Such an adaptation, however, occurred only when other cues for distance, cues that represented objective distances correctly, were given along with the altered oculomotor cues. Under conditions where all other cues were absent and only the altered oculomotor cues represented the distances of visual objects, no adap- 
tation took place (Wallach \& Halperin, 1977). Thus, adaptation in distance perception belongs to a type of adaptation process that depends on the presence of veridical cues. This type of adaptation occurs where several different cues represent the same objective condition (e.g., distance) and determine the same perceived property (perceived distance). We call such a combination of cues "paired cues." When one or two, but not all, of such a combination of cues are altered by spectacles or other interposed devices, a cue discrepancy results that may bring about adaptation. $^{2}$

Paired cues, of course, give rise to different perceptual processes. Each of these processes, however, determines the quantitative aspect of the same perceptual property in which they all result. Normally, when paired cues represent an objective condition, the different perceptual processes they evoke result in the same value for that single perceptual property. But when one or two of the cues are altered by an interposed device, the equivalence of all the paired cues is lost. At the same time, the perceptual processes that evaluate these cues operate as before. Therefore, the perceptual property that results from these processes will have different values, dependent on the particular cue from which it originates. ${ }^{3}$ This value difference causes the adaptation. When the evaluation process connected with the altered cues is so changed that the value difference is diminished, it will partly compensate for the effect of the cuealtering device. In this type of adaptation, the developing compensation diminishes the effect of a cue discrepancy by altering cue evaluation. It follows that a veridical cue also may undergo a change in evaluation as a result of prolonged exposure to a cue discrepancy. Wallach and Frey (1972b) introduced the term "counteradaptation" for such a change and reported one; it will be described below in the Discussion section. Wallach and Huntington (1973) obtained simultaneous changes in the evaluation of a veridical cue and of an altered one in connection with exposure to a wedge prism. ${ }^{4}$

Adaptation based on cue discrepancy is a valuable tool in perception research. It can be used to verify the effectiveness of potential perceptual cues. Wallach, Frey, and Bode (1972) have used adaptation in distance perception based on oculomotor cues to demonstrate the effectiveness as a distance cue of the image size of familiar objects. They illuminated, in a darkened room, three different familiar objects, one at a time, with a narrow light beam while subjects watched, wearing either the "near glasses" or spectacles with the opposite effect. In both cases, a changed evaluation of the oculomotor cues developed that partially compensated for the particular effect of the spectacles that were worn. Since only the familiar objects were visible while the subjects wore the spectacles, their image sizes were the only potential distance cues present in addition to the altered oculomotor cues. In tests where only oculomotor cues were available, a change in estimated size, ranging from $15 \%$ to $20 \%$ for different adaptation and test conditions, demonstrated adaptive changes in registered distance in corresponding amounts.

In addition to these size estimation tests, Wallach, Frey, and Bode gave depth estimation tests before and after the adaptation period. Like perceived size, perceived depth is a function of registered distance. This is so because the retinal disparity with which an objective depth interval is given decreases with the observation distance. Therefore, distance is taken into account in the process that connects retinal disparity with perceived depth. In other words, there is a stereoscopic depth constancy that resembles size constancy, the process that connects image size with perceived size and takes distance into account. The two constancies differ quantitatively, because the image size of an object is inversely proportional to the first power of distance, while retinal disparity is inversely proportional to the square of the distance. Therefore, while perceived size is proportional to the product of image size and registered distance, perceived depth should be proportional to disparity times the square of registered distance if perceived depth is to correspond to objective depth. Up to a distance of $200 \mathrm{~cm}$, this is approximately the case. ${ }^{5}$

Because perceived depth varies more with registered distance than perceived size, a change in registered distance due to adaptation should have a greater effect on depth estimates than on size estimates. This was found to be true. The experiment showed that the image sizes of familiar objects serve as distance cues that are just as directly effective as oculomotor cues. This contradicted the view that judgments of distances of familiar objects are derived from their image sizes by an inferential process.

Our demonstration of the effectiveness of HMP as a cue for distance was an analogue of the experiment just described. The experiment tested the effectiveness of head-movement parallax (HMP) by employing it as the sole veridical distance cue in adaptation to near glasses. In such an experiment, accommodation operates on the nonveridical side of the cue discrepancy that leads to adaptation, and its effect cannot confound the effect of head-movement parallax. During the exposure period, the target was at a distance of $60 \mathrm{~cm}$ from the eyes and the spectacles caused the eyes to be accomodated and converged for a distance of $31.2 \mathrm{~cm}$ when they focused on the target at $60 \mathrm{~cm}$ distance. This, then, was the cue discrepancy that prevailed during the exposure period: while the eyes were accomodated and converged for a target distance of $31.2 \mathrm{~cm}$, movement parallax, if it were effective, would represent the target as being at $60 \mathrm{~cm} .6$ If exposure to this discrepancy caused an adaptation, that is, caused a 
change in the evaluation of accommodation and convergence such that they represented distances as larger than before exposure, it would show that the distances of 60 and $31.2 \mathrm{~cm}$ could be discriminated by means of HMP.

McConkie and Farber had used lateral head movements, but our subjects caused the parallax by head turning, which also displaces the eyes laterally. We believe HMP to be a learned distance cue. Since the extent of head movement has to be taken into account when distances are discriminated on the basis of parallax, it is quite possible that HMP operates as a distance cue in relation to specific kinds of head movement rather than to displacements of the eyes independently of the movements by which they are produced. Since head turning occurs much more frequently than lateral head movement, a parallax cue connected with turning may be learned better or it may be used by more subjects. We therefore had our subjects turn their heads during the exposure period.

Our experiment was done with two groups of subjects. The experimental subjects (Group E) wore the near glasses and turned their heads during the exposure period. The subjects of the control group (C) also wore the near glasses during the exposure period, but their heads were kept stationary. Both groups were tested, without glasses, for a changed evaluation of oculomotor cues with two different measures of registered distance; they gave size and depth estimates before and after the exposure period.

\section{METHOD}

\section{Subjects}

The subjects were male and female undergraduate students. Twenty served in Group E and 14 in Group C.

\section{Apparatus and Procedure}

The near glasses that the subjects wore during the exposure period were described by Wallach and Frey (1972a). These spectacles caused the eyes to be accommodated and converged for a distance of $31.2 \mathrm{~cm}$ when the actual target distance was $60 \mathrm{~cm}$. During the exposure period, which lasted $20 \mathrm{~min}$, every subject watched a TV broadcast of his choice on a set with a $12 \mathrm{~cm}$ screen that was placed straight in front of him and $60 \mathrm{~cm}$ from his eyes. The TV picture was of low brightness and the only object visible. The subjects of Group E wore a welder's headgear that was attached to a vertical shaft above the subject's seat in such a way that the shaft coincided with the vertical rotation axis of his head. Elastic stops limited the rotation of the shaft and of the attached head to $20^{\circ}$ to either side of the forward direction. A switch in each stop activated a counter. Turning the head through an angle of $40^{\circ}$ caused a horizontal displacement of the eyes of about $7 \mathrm{~cm}$. During the 20-min exposure period, the subjects of Group $\mathrm{E}$ made, on the average, 1,039 head turns. In the case of the control subjects, the head was kept motionless in position by a bite mold.

All subjects made their size and depth estimates from another seat to which they were led in the dark. There they saw a small wire shape, a pyramid, which was exposed in such a way that only oculomotor cues for its distance were available. The pyramid's square base was vertical and faced the subject, and its axis coincided with his line of sight. The pyramid was mounted in an aperture in the front side of a large black box, with its base in the plane of the aperture. It was visible against a rearilluminated translucent sheet inside the box. The perceived size of the pyramid was represented by an estimate of the length of the diagonal of the pyramid base, and an estimate of the length of the pyramid's axis served as the depth estimate. The base of the pyramid was $66.7 \mathrm{~cm}$ from the eyes of the subject, whose head was fixed in a head- and chinrest. The subject gave his estimates by selecting and adjusting the length of a small metal rod, using the sense of touch only. These tests were identical with those used by Wallach, Frey, and Bode, except that only the larger one of the two pyramids they used was employed by us. ${ }^{7}$ Half of the subjects in each group first gave a size estimate followed by a depth estimate; for the other half, the sequence was reversed.

\section{RESULTS}

Table 1 lists the mean size estimates and the mean depth estimates that were obtained before and after exposure, along with the objective measurements of the pyramid. For Group E, the mean size and the mean depth estimates were larger after the exposure period, and each of these differences was highly significant $(p<.001)$. The increase in size of the estimates implied an increase in registered distance. Such an increase partially compensated for the effect of the spectacles. For the control group, there were small increases in the mean size and mean depth estimates, one of which, the increase in depth estimation, was significant at the .05 level. ${ }^{8}$ But the mean changes in estimated size and depth were much larger for Group E than for Group C, and the difference between these changes was highly significant. In the case of size, the mean changes differed by $1.43 \mathrm{~cm}$, and this difference was significant at better than the .001 level, with $\mathrm{t}(32)=$ 3.57. The two mean depth changes differed by $1.84 \mathrm{~cm}$, with this difference significant at better than the .01 level, with $t(32)=2.59$. The presence of HMP during the exposure period produced a strong adaptation effect, which, where size estimates were concerned, matched the effects that Wallach, Frey, and Bode (1972) obtained in their Experiments 4 and 5 , where perspective or image size of familiar

Table 1

Mean Size and Depth Estimates in Centimeters Before and After Exposure to Near Glasses, With or Without Head Movement Parallax (HMP)

\begin{tabular}{lccccc}
\hline & \multicolumn{2}{c}{$\begin{array}{c}\text { E, With HMP } \\
(\mathrm{N}=20)\end{array}$} & & \multicolumn{2}{c}{$\begin{array}{c}\text { C, Without HMP } \\
(\mathrm{N}=14)\end{array}$} \\
\cline { 2 - 3 } \cline { 5 - 6 } & Size & Depth & & Size & Depth \\
\hline Objective & 11.00 & 10.00 & & 11.00 & 10.00 \\
Estimates & & & & \\
$\begin{array}{l}\text { Preexposure } \\
\text { Postexposure }\end{array}$ & 10.97 & 12.09 & & 9.53 & 10.77 \\
Difference & 1.90 & 14.61 & & 10.03 & 11.46 \\
Change* & 17.6 & 2.52 & & .50 & .69 \\
& & 20.8 & & 5.2 & 6.4 \\
\hline
\end{tabular}

*Proportional change (expressed as percentages). 
objects, respectively, served as veridical distance cues.

\section{DISCUSSION}

Our experiment showed that HMP can function as the veridical cue to absolute distance in a cue discrepancy that leads to sizable and highly significant changes in the evaluation of convergence and accommodation as cues for distance. Since the cue discrepancy involved the distances of 60 and $31.2 \mathrm{~cm}$, HMP must have provided a discrimination of these distances. ${ }^{9}$ This conclusion makes it likely that whatever adequate distance matches McConkie and Farber (Note 1) obtained were due to HMP rather than to accommodation. Apart from demonstrating effectiveness of HMP as a distance cue, our experiment adds to the work of Wallach, Frey, and Bode (1972), who demonstrated that perspective cues and the image sizes of familiar objects can serve as the sole veridical distance cues in adaptation in distance perception based on oculomotor cues. We know now that HMP can fill the same role.

Our experiment is an example of the technical use that can be made of adaptation. Adaptation provided a test of the effectiveness of a potential cue, HMP, in the presence of an extraneous cue, accommodation. By having accommodation operate on the other side of the cue discrepancy that caused the adaptation, we avoided having the effect of HMP contaminated by the ever present accommodation.

There is still another way in which adaptation can be used to deal with extraneous cues. Instead of having the extraneous cue serve as the altered cue, as was done in our experiment, the cue whose effectiveness is to be demonstrated can serve as the altered cue in the cue discrepancy that causes adaptation. Functioning as the altered cue and therefore as nonveridical, the potential cue is isolated against any extraneous cue, known or unknown. An experiment that was actually performed may serve as an example. It will be outlined here because control of extraneous cues was not among its purposes; this aspect was not even mentioned in the original presentation.

Wallach and Frey (1972b) set out to show that the increase in the size of the retinal image of an approaching object can serve as the sole cue that causes a change in the evaluation of oculomotor cues. They asked whether an object that alternately moved toward and away from the subject between the distances of 400 and $33.3 \mathrm{~cm}$ would convey sufficient distance information to cause adaptation to "near glasses." Such spectacles may, for instance, alter the oculomotor cues in such a way that the actual far and near points of that motion path would be given with oculomotor adjustments for 80 and $25 \mathrm{~cm} .{ }^{10} \mathrm{~A}$ motion path ranging between the latter distances would cause the image size to change by a factor of 3.2, whereas the actual motion path between 400 and $33.3 \mathrm{~cm}$ would involve an image size change by a factor of 12 . Would the discrepancy between the latter image size change and the altered oculomotor cues that correspond to the smaller distance change produce an adaptation?

This experiment was actually performed without the use of spectacles. The motion path actually ranged between 80 and $25 \mathrm{~cm}$ from the subject's eyes, and the objective size of the moving object, a luminous diamond-shaped surface, varied so that it simulated a motion path between the distances of 400 and $33.3 \mathrm{~cm}$ from the eyes. The size of the diamond increased as it traveled toward the subject and decreased as it receded, in such a way that its retinal image underwent the changes that the longer motion path would produce.

Observing this contracting and expanding figure move back and forth for 20 min resulted in a mean increase of size perceived on the basis of oculomotor cues in the amount of $10 \%$ when the distance of the test figure was $33.3 \mathrm{~cm}$ and in a size increase of $16 \%$ when the test distance was $66.7 \mathrm{~cm}$, indicating that corresponding changes in registered distance had taken place. There was also a test for apparent distance where the subject pointed at a target line from the side; it yielded a mean distance increase of $11 \%$ when the target line was $33.3 \mathrm{~cm}$ from the eyes. All these changes were highly significant. They occurred even though, in the exposure period, oculomotor cues represented distances veridically. In fact, the purpose of the experiment was to demonstrate that adaptation might consist in a change away from veridicality. However, simulating the larger motion path with objective size changes and allowing the oculomotor cues to represent objective distances has the advantage that other distance cues that might have been present could not represent the larger motion path. The image size cues that did represent it would assuredly operate in isolation. Thus, the technique used in this experiment, that of employing veridically those cues that are to be changed by adaptation and of altering the cue whose effectiveness is put to a test, deals effectively with the problem of extraneous cues. Any extraneous cues that might have operated during the exposure period would tend to prevent rather than aid adaptation.

\section{REFERENCE NOTE}

1. McConkie. A..\& Farber. J. Monocular head movement parallux and absolute distance perception. Unpublished manuscript. Cornell Liniversity, 1978.

\section{REFERENCES}

Ebenholtz, S. M., \& Wolfson. D. M. Perceptual aftereffects of sustained convergence. Perception \& Psychophysics, 1975, 17, 485.491. 
Johansson, G. Monocular movement parallax and near-space perception. Perception, 1973, 2, 135-146.

ONo, H., \& Comerford, J. Stereoscopic depth constancy. In W. Epstein (Ed.), Stability and constancy in visual perception. New York, N.Y: Wiley-Interscience Publication, 1977.

WaLLACH, H. Informational discrepancy as a basis of perceptual adaptation. In S. J. Freedman (Ed.), The neuro-psychology of spatially oriented behavior. Homewood, Ill: Dorsey Press, 1968.

W ALlACh. H. On perception. New York, N.Y: Quadrangle/The New York Times Book Co., 1976.

Wallach, H., \& FreY, K. J. Adaptation in distance perception based on oculomotor cues. Perception \& Psychophysics, 1972, 11, 77-83. (a)

W Allach, H., \& FREY, K. J. On counteradaptation. Perception \& Psychophysics, 1972, 11, 161-165. (b)

W allach, H., Frey, K. J., \& Bode, K. A. The nature of adaptation in distance perception based on oculomotor cues. Perception \& Psychophysics, 1972, 11, 110-116.

Wallach, H., \& Halperin, P. Eye muscle potentiation does not account for adaptation in distance perception based on oculomotor cues. Perception \& Psychophysics, 1977, 22, 427-430.

Wallach, H., \& Huntington, D. Counteradaptation after exposure to displaced visual direction. Perception \& Psychophysics, 1973, 13, 519-524.

\section{NOTES}

1. Johansson (1973) found head-movement parallax an effective distance cue up to a distance of $240 \mathrm{~cm}$. However, he deliberately enriched the experimental conditions with "perspective transformation due to parallactic movements and the manifolding this implies." In what manner this "enrichment" contributed to the distance information that head-movement parallax provided was not analyzed.

2. Other kinds of adaptation that belong to this group are adaptation in stereoscopic depth perception, adaptation to displaced visual direction, and adaptation to visual tilt. See Wallach (1976) chapter 10.

3. When all cues operate simultaneously, the immediate effects of such interposed devices differ widely, dependent on the device and the perceptual property involved. The altered cue may determine what is perceived or the other cue or cues that represent the objective conditions correctly may do so, or there will be a compromise. See Wallach (1968) pp. 211-221 or Wallach (1976) pp. 238-250.
4. We actually tested for counteradaptation in connection with the present experiment. Unfortunately, no decisive test was possible. Instead, we tested for a particular manifestation of counteradaptation. The negative result we obtained was inconclusive, because it did not prove that there was no counteradaptation.

5. For the evidence on stereoscopic depth constancy, see Ono and Comerford (1977).

6. Walter $C$. Gogel suggested to us that the spectacles that the subjects wore during the adaptation period also change the parallax with which the target is given and increase the motion-parallax distance. We found that this was correct. The spectacles consisted of a combination of wedge prisms and -1.5-diopter lenses. Because there is a distance between the lens and the eye, the lenses operate as minifiers of small power. With the distance between the eyes and the lens about $3 \mathrm{~cm}$, the lenses diminish visual angles by about $5 \%$ and displace visual directions during a turning of the head in the same proportion. This results in an altered parallax such that a target at the distance of $60 \mathrm{~cm}$ will be given with a parallax that corresponds to a target distance of about $85 \mathrm{~cm}$.

7. We omitted the smaller pyramid because Wallach, Frey, and Bode had found that its depth was grossly overestimated.

8. The reason for this small effect is not clear. It seems possible that eye-muscle potentiation caused by the steady converging of the eyes for a fixed distance could have produced the effect. This explanation would make it necessary to assume that for a majority of our subjects the spectacles produced convergence of the eyes for a distance a few centimeters shorter than $30 \mathrm{~cm}$, the balance point for eye-muscle potentiation determined by Ebenholtz and Wolfson (1975). This is possible because the distance cue provided by the convergence change caused by our glasses varies with interocular distance.

9. As was explained in Note 6, the spectacles had an effect on HMP and increased the target distance represented by HMP from $60 \mathrm{~cm}$ to about $85 \mathrm{~cm}$. This raised the cue discrepancy produced by the spectacles by one-third, from the equivalent of 1.5 lens diopters to 2 lens diopters.

10. Such spectacles would consist of -1-diopter spherical lenses and of prisms that change each eye's line of sight by an angle of $1.85^{\circ}$. They would force the eyes to view objects at every distance with additional accommodation and convergence in these amounts.
(Received for publication April 3, 1978; revision accepted September 29, 1978.) 